\title{
Evaluation of two commercial jet nebulisers and three compressors for the nebulisation of antibiotics
}

\author{
J C C Hung, G Hambleton, M Super
}

\begin{abstract}
Nebulised antibiotics have been shown to be beneficial in the treatment of lung infections in cystic fibrosis. Studies on the efficiency of nebuliser systems are constantly required in view of the large number of compressor/drug/nebuliser combinations which are possible and the development of new systems and drugs. Six combinations of three commercially available compressors were compared (PortaNeb 50 (Medic-Aid; 5.4-6.1 1/min), Turboneb (Medix; 8.3-9.1 $1 / \mathrm{min}$ ), and CR 60 (Medic-Aid; 7·3-7.8 $1 / \mathrm{min}$ )) and two jet nebulisers (Microneb III (Lifecare) and System 22 Acorn (Medic-Aid)) for the nebulisation of colomycin, gentamicin, and ciprofloxacin. Aerosol droplet size, nebulisation time, and aerosol output were determined. Turboneb and CR 60 reduced the nebulisation time and produced higher proportions of 'respirable' $(<5 \mu \mathrm{m}$ diameter) antibiotic aerosols. The residual volume of the Microneb III was lower than that of the System 22 Acorn. It was found that the Turboneb and CR 60, when coupled with either Microneb III or System 22 Acorn, were suitable for the nebulisation of all three antibiotics. Of the equipment tested, Turboneb coupled with Microneb III was the most efficient combination. Even with this combination, only around $50 \%$ of the nominal dose was released as respirable aerosol.

(Arch Dis Child 1994; 71: 335-338)
\end{abstract}

Jet nebulising systems have been used to deliver drugs to the lungs for many years. There is a wide range of drugs, ${ }^{1}$ particularly in the treatment of cystic fibrosis, which are required to be delivered by nebulisation; their physicochemical properties are diverse. It is important to assess nebuliser performance against each therapeutic drug to maximise efficacy and to minimise wastage. ${ }^{2} 3$

The use of antibiotics as aerosols has become routine in the treatment of pulmonary infection in cystic fibrosis, where it has been shown to be of benefit in pseudomonas infection. ${ }^{4-6}$ There is much published information $^{78}$ on the use of jet nebulising systems, but studies $^{9-13}$ such as these are constantly required in view of the large number of compressor/drug/nebuliser combinations which are possible and the development of new systems and drugs.
We studied three compressors used in combination with two jet nebulisers to determine their suitability for use with three antibiotics with different osmolarities. The equipment is currently used in our hospital. The compressors were chosen for their ability to deliver different air flows to the nebulisers and the jet nebulisers for their different designs for example, jet collar tube and reservoir shape.

\section{Materials and methods}

Of the three antibiotics tested, two were ready to use solutions (gentamicin $(80 \mathrm{mg} / 2 \mathrm{ml}$; Roussel) and ciprofloxacin (2 mg/ml; MSD)) and one was a powdered preparation (colomycin (1 MU or $80 \mathrm{mg}$; Pharmax)). Four millilitres ${ }^{23}$ of gentamicin or ciprofloxacin were used, and the coloymcin was diluted with $4 \mathrm{ml}$ of normal saline. Six samples of each antibiotic solution were nebulised with six combinations of two jet nebulisers (Microneb III (Lifecare) and System 22 Acorn (Medic-Aid)) and three compressors (CR 60 (Medic-Aid), Turboneb (Medix), and PortaNeb 50 (Medic-Aid)). The nebuliser pressure-flow characteristics were measured by attaching a 'ball in a column' air flow meter (Oxylitre) and a Bourdon type pressure gauge (Oxylitre) via a $T$ connector interposed between the nebuliser and compressed air. Measurements were made with six samples of each type of nebuliser.

Aerosol droplet size was measured for each nebuliser/compressor combination by a Malvern Instruments 2600 HSD laser particle and droplet analyser. The particle size was derived from the laser diffraction angles, based on the Fraunhofer theory, by an on-line computer. The size distribution of the aerosol mass was plotted into 15 size bands from $1 \cdot 22$ to $118 \mu \mathrm{m}$ on a log-normal scale. The mass median diameter is the diameter around which the aerosol mass is equally divided (that is, half of the aerosol mass is contained in particles smaller and half of the aerosol mass in particles larger than the mean mass diameter). The percentage of the aerosol mass contained in droplets less than $5 \mu \mathrm{m}$ in diameter $\left(P_{5}\right)$ was calculated by the computer from the cumulative percentage plot.

The nebulisers were fitted with their dedicated $T$ shaped mouthpieces and the filters were attached at the opposite end of the outlets. They were clamped onto the particle sizer with the tip of the mouthpieces at $2.5 \mathrm{~cm}$ from the laser beam. The aerosol spray was vented away via a funnel using a suction pump. 
Mean (SEM) dynamic pressure and flow values of the compressors when coupled with either the Microneb III or System 22 Acorn nebulisers

\begin{tabular}{|c|c|c|c|c|}
\hline \multirow[b]{2}{*}{ Compressor } & \multicolumn{2}{|l|}{ Microneb III } & \multicolumn{2}{|c|}{ System 22 Acorn } \\
\hline & $\begin{array}{l}\text { Pressure } \\
\text { (psi) }\end{array}$ & $\begin{array}{l}\text { Flow } \\
\text { (Lmin) }\end{array}$ & $\begin{array}{l}\text { Pressure } \\
\text { (psi) }\end{array}$ & $\begin{array}{l}\text { Flow } \\
\text { (U/min) }\end{array}$ \\
\hline $\begin{array}{l}\text { Turboneb } \\
\text { CR } 60 \\
\text { PortaNeb } 50\end{array}$ & $\begin{array}{l}34(0.04) \\
26(0 \cdot 06) \\
17 \cdot 1(0.05)\end{array}$ & $\begin{array}{l}9 \cdot 1(0.08) \\
7 \cdot 8(0.03) \\
6 \cdot 1(0.08)\end{array}$ & $\begin{array}{l}35 \cdot 1(0.03) \\
27 \cdot 2(0.02) \\
17 \cdot 7(0.49)\end{array}$ & $\begin{array}{l}8.3(0.04) \\
7.3(0.01) \\
5.4(0.59)\end{array}$ \\
\hline
\end{tabular}

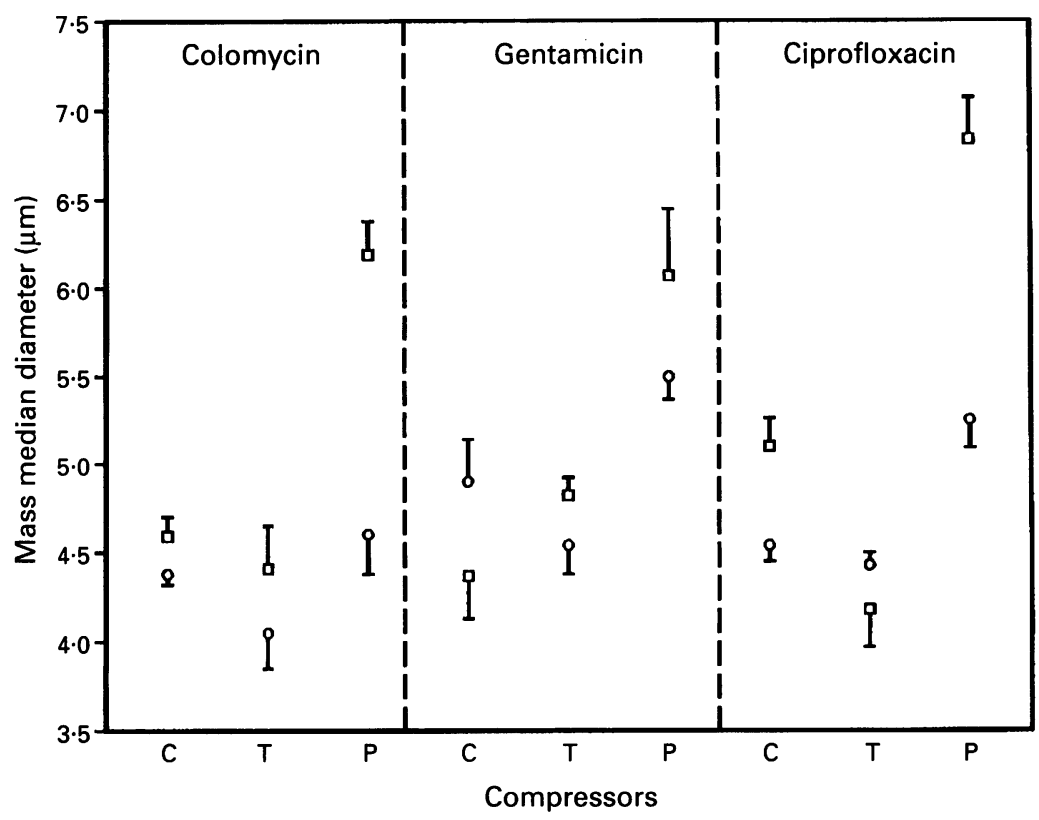

Figure 1 Mean (SEM) mass median diameters for different combinations of compressors ( $C=C R$ 60; $T=$ Turboneb; $P=$ PortaNab 50) and nebulisers (squares=System 22; circles $=$ Microneb III).

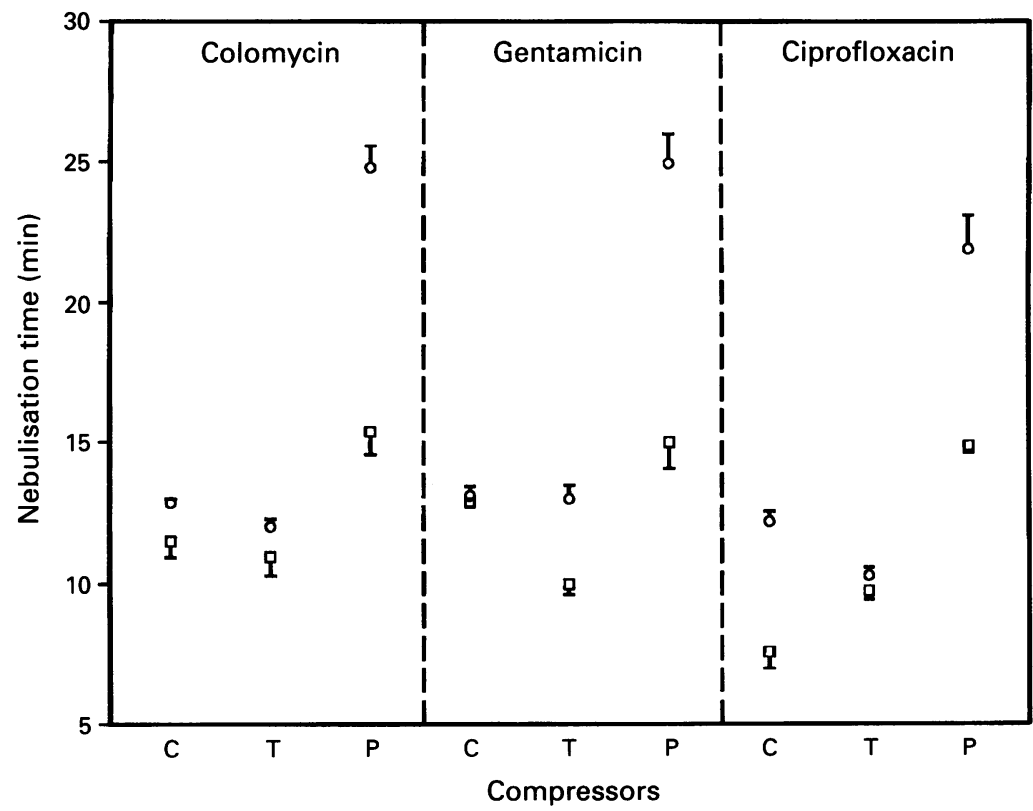

Figure 2 Mean (SEM) nebulisation times for different combinations of compressors $(C=C R$ 60; $T=$ Turboneb; $P=$ PortaNeb) and nebulisers (squares $=$ System 22 ; circles $=$ Microneb III). as described by Newman et al. ${ }^{913}$ The osmolarity was measured with an osmometer (Advanced Instrument). The percentage of dose released as aerosol was given by:

((mass of drug released as aerosol)/(nominal drug dose $)) \times 100 \%$

The mass of drug released as aerosol was calculated as

$$
\frac{M_{i} C_{i}}{D_{i}}-\frac{M_{r} C_{r}}{D_{r}}
$$

where $M_{i}$ and $M_{r}$ were the mass of drug solution initially placed in the nebuliser reservoir and the residual mass of drug solution after nebulisation respectively, $C_{i}$ and $C_{r}$ were the initial and final drug concentrations, and $\mathrm{D}_{i}$ and $D_{r}$ the initial and final solution densities.

The results were analysed using the Wilcoxon rank sum test and the significant level was taken at $\mathrm{p}<0 \cdot 05$.

\section{Results}

The table gives the mean and standard error of the mean (SEM) dynamic pressure and flow values for each compressor/nebuliser combination. For each compressor, there was a significant difference $(p<0.05)$ in the values when combined with either nebuliser.

The respective mean (SEM) osmolarities (36 samples each) of colomycin, gentamicin, and ciprofloxacin are $347.9(1.14), 174.8$ $(0.98)$, and $294.6(0.31)$ mosmo/l.

Figures 1-4 show the results for ciprofloxacin and gentamicin. With both nebulisers, the CR 60 and Turboneb produced significantly $(\mathrm{p}<0.05)$ smaller mean mass diameter values. For both nebulisers, the $C R$ 60 and Turboneb significantly $(p<0.05)$ reduced the nebulisation time compared with the PortaNeb 50. The nebulisation times were significantly $(p<0.05)$ longer with the Microneb III. The different flow rates of the three compressors did not significantly affect the residual volume in either nebuliser. The Microneb III, however, had a significantly $(p<0.05)$ lower residual volume than the System 22 Acorn.

The mass of both antibiotics released as aerosols did not vary significantly among the three compressors when used with either nebuliser (fig 4). The mass of antibiotics contained in 'respirable' $(<5 \mu \mathrm{m}$ in diameter $)$ droplets was significantly greater $(p<0.05)$, however, when either nebuliser was used with the CR 60 and Turboneb. The Microneb III released significantly $(p<0.05)$ more aerosol and $P_{5}$ than the System 22 Acorn.

The range of volumes produced by the addition of $4 \mathrm{ml}$ saline to $80 \mathrm{mg}$ of colomycin was $3 \cdot 8-4 \cdot 2 \mathrm{ml}$. Figures $1-4$ also show the results for colomycin. With the System 22 Acorn, the CR 60 and Turboneb produced significantly $(p<0.05)$ lower $T$, higher $P_{5}$, and smaller mean mass diameter values than the PortaNeb 50. With the Microneb III, the Turboneb produced significantly lower $T$ and higher $P_{5}$ values than the CR 60 and PortaNeb 50 . There was no significant difference in the compressor to the The solution masses were measured by weighing. The residual volume $\left(\mathrm{V}_{\mathrm{r}}\right)$ drug concentration was calculated by comparing the osmolarity of the initial and the final solutions,

The antibiotic solutions were nebulised until no visible aerosol was released for 15 seconds. The nebulisation time (T) was measured by

Hod




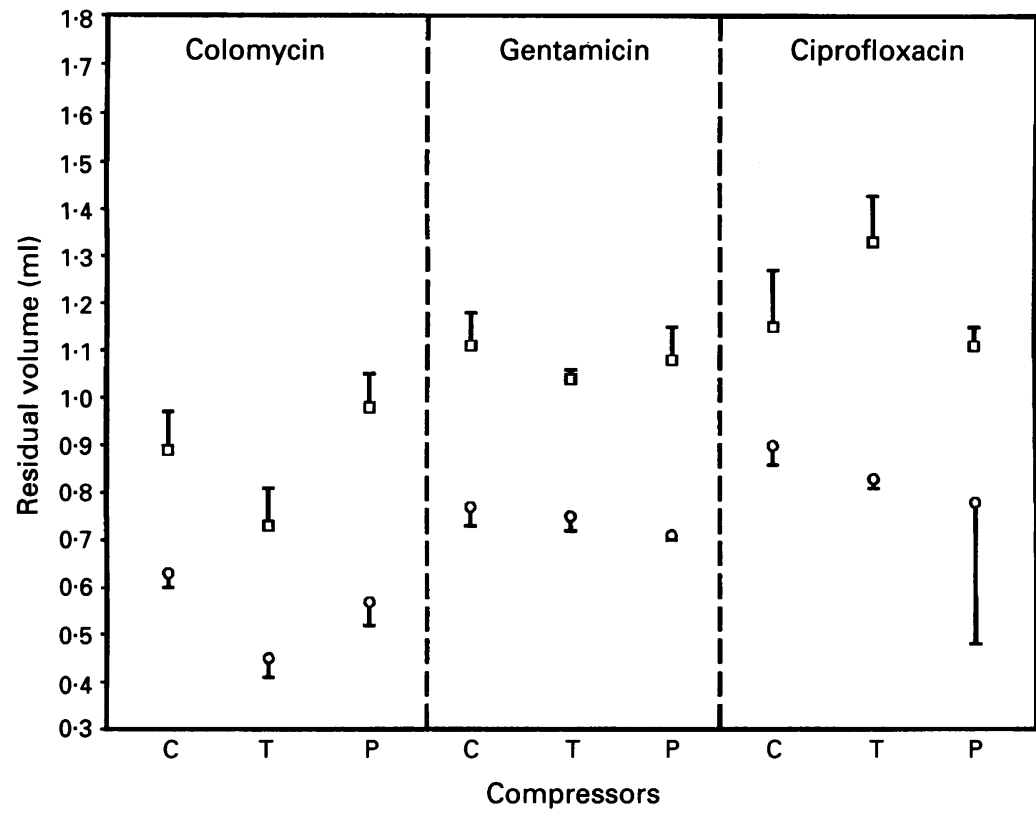

Figure 3 Mean (SEM) residual volumes for different combinations of compressors ( $C=C R$ 60; $T=$ Turboneb; $P=$ PortaNeb) and nebulisers (squares $=$ System 22 ; circles= Microneb III). osmolarity and the percentage of drug released. Our results agree well with those of Smye and Littlewood, ${ }^{10}$ however, that a higher percentage of colomycin, with a lower surface tension, is released than gentamicin. The variable effect of the tonicity of the colomycin solution on the lung function of patients with cystic fibrosis is highlighted in a study by Dodd et al. ${ }^{14}$

The nebulisation times were shorter for the System 22 Acorn; this was partly because its residual volume was larger. It has been shown that the output efficiencies of nebulisers decrease sharply during the intermittent phase. ${ }^{2}{ }^{15}$ Although the solutions become more concentrated towards the end, most of the drug is released in the initial part of nebulisation. ${ }^{15}$ It may be possible to limit the nebulisation time with the Microneb III, which has a longer linear phase, and still achieve a similar total drug output to the System 22 Acorn. Further studies are needed to assess the optimum duration of nebulisation for the antibiotics with each nebulising system.

Smye et al ${ }^{16}$ showed that the reduction of the nebuliser internal surface area improves the efficiency of the nebuliser. This agrees well with our findings. The conical base in the well of the Microneb III, in contrast with the flat base in the System 22 Acorn, allows less accumulation of drug solutions at the base of the nebuliser and produces a smaller residual volume. The inverted umbrella shaped jet collar tube of the System 22 Acorn probably also contributes to the increase in residual volume by trapping fluid on its surfaces. For the same reasons, tilting of the nebuliser, towards the end of nebulisation, is more important for the System 22 Acorn than the Microneb III. As the residual volumes of the nebulisers, for the three antibiotics, are not dependent on the power of the compressors, the design of the nebulisers is crucial to maximise the release of drug solutions.

An aerosol suitable for inhalation should have a mean mass diameter of less than $5 \mu \mathrm{m} .{ }^{7817}$ When used with the more powerful CR 60 or Turboneb, both nebulisers produced mean mass diameters of less than $5 \mu \mathrm{m}$. The weaker PortaNeb 50, except when used with the Microneb III to nebulise colomycin, produced unacceptably large mean mass diameters with either nebuliser and a low proportion of respirable aerosol droplets. The overall performance of the Microneb III was better than that of the System 22 Acorn. This was more apparent when coupled with the weaker PortaNeb 50.

Froth in the nebuliser, especially with colomycin, forms bridges between the top and base and hastens the return of the solutions from the top to the bottom. Excessive frothing, however, causes an irregular output, increases the residual volume, and produces an unpredictable particle size.

Excipients can alter the properties of the drug solutions and, hence, affect the performance of the delivery system. The reduction of surface tension of the antibiotic solution improves the performance of nebulisers. ${ }^{16}$
Figure 4 Mean percentages of dose released (solid lines) and dose in $<5 \mu \mathrm{m}$ droplets (dashed lines) for different combinations of compressors ( $C=C R$ 60; $T=$ Turboneb; $($ dashed lines) for different combinations of compressors $(C=C R 60 ; T=T$.
$P=$ PortaNeb) and nebulisers (squares $=$ System 22; circles=Microneb III). 
Artificial and natural surfactants are safe and non-immunogenic in the treatment of hyaline membrane disease. ${ }^{18}$ In a pilot study with Curosurf, it was found that it reduces the amount of droplet formation on the interior surfaces of the nebulisers, and reduces the residual volumes of the nebulisers; however, the nebulisation time is prolonged as more of the drug solution becomes available for nebulisation. Studies are being planned to assess the use of surfactant in patients with cystic fibrosis.

The effects of other excipients (for example, hydroxbenzoate in gentamicin and phenol in tobramycin) present in the intravenous preparations on the lungs are still unknown. Preparations that are safe for intravenous use may not necessarily be safe when given by inhalation, especially when high concentrations of the active ingredients and the excipients are achieved in the lungs. It appears that formulations should be developed specifically for inhalation purposes.

In agreement with previous studies on compressors of different power outputs, ${ }^{9-12}$ we conclude that ciprofloxacin, colomycin, and gentamicin are adequately nebulised by the $C R$ 60 or Turboneb, with the Microneb III or System 22 Acorn. The PortaNeb 50 is not suitable for the nebulisation of antibiotics. The design of the nebuliser can improve the efficiency of the weaker compressor, however. Studies are required to assess the effects of various compressor flow rates on the newer designs of nebulisers (for example, the Sidestream (Medic-Aid), Ventstream (MedicAid), Pari LC Plus (PARI) and Pari LL (PARI)), which enhance drug output using the extra Venturi effect. Of the equipment tested, the best results were obtained when the Turboneb was used with the Microneb III. Even with this combination, only about $50 \%$ of the nominal dose was released as respirable aerosols. Extrapolating to emerging preparations - for example, rhDNase - likely to be administered by inhalation, it is clear that the efficiency of the delivery system will require specific evaluation using similar methods.

Dr J C C Hung is supported by the Cystic Fibrosis Research Trust and Merck Ltd. We thank Dr J Lim for his helpful advice and Fisons plc, $\mathrm{R}$ and $\mathrm{D}$, for the use of the Malvern particle analyser.

1 Clarke SW, Newman SP. Therapeutic aerosols 2 - drug available by the inhaled route. Thorax 1984; 39: 1-7.

Clay M, Pavia D, Newman SP, Lennard-Jones T, Clark SW. Assessment of jet nebulisers for lung aerosol therapy. Lancet 1983; 2: 592-4.

3 Clay M, Pavia D, Newman SP, Clarke SW. Efficiency of je nebulisers in the production of therapeutic aerosols. Thorax 1982; 37: 788

4 Hodson ME, Penketh AR, Batten JC. Aerosol carbenicillin and gentamicin treatment of Pseudomonas aeruginosa infection in patients with cystic fibrosis. Lancet 1981; ii: 1137-9.

5 Littlewood JM, Miller MG, Ghoneim AT, Ramsden CH. Nebulised colomycin for the early Pseudomonas aeruginosa colonisation cystic fibrosis. Lancet 1985; ii: 865 .

6 Jensen T, Pedersen SS, Garne S, Heilmann C, Hoiby N, Koch C. Colistin inhalation therapy in cystic fibrosis patients with chronic Pseudomonas aeruginosa lun infection. F Antimicrob Chemother 1987; 19: 831-8.

7 Newman SP, Clarke SW. Therapeutic aerosols 1 - physica and practical considerations. Thorax 1983; 38: 881-6.

8 Littlewood JM, Smye SW, Cunliffe H. Aerosol antibiotic treatment in cystic fibrosis. Arch Dis Child 1993; 68: 788-92.

9 Newman SP, Pellow PG, Clarke SW. Efficient nebulisation of powdered antibiotics. Int $\mathcal{F}$ Pharm 1987; 36: 55-60.

10 Smye SW, Littlewood J. The performance of fou commercial nebuliser systems [abstract]. Abstracts in 18th European Cystic Fibrosis Conference. 1993: 120.

11 Newman SP, Pellow PG, Clarke SW. Choice of nebulisers and compressors for delivery of carbenicillin aerosol. Eur $\mathcal{F}$ Respir Dis 1986; 69: 160-8.

12 Newman SP, Pellow PG, Clarke SW. The flow-pressure characteristics of compressors used for inhalation therapy Eur $\mathcal{f}$ Respir Dis 1987; 71: 122-6.

13 Newman SP, Pellow PG, Clay MM, Clarke SW. Evaluation of jet nebulisers for use with gentamicin solution. Thora 1985; 40: 671-6.

14 Dodd M, Maddison J, Abbott J, Webb AK. The effect of the tonicity of nebulised colistin on lung function in adults with cystic fibrosis [abstract]. Cystic Fibrosis Conference. 1993: 121

15 O'Callaghan C, Clark AR, Milner AD. Why nebulise for more than five minutes? Arch Dis Child 1989; 64: 1270-3.

16 Smye SW, Shaw A, Norwood HM, Littlewood JM. Some factors influencing the efficiency of a jet nebuliser system factors influencing the efficiency of a jet n

17 Task Group on Lung Dynamics. Deposition and retention models for internal dosimetry of the human respiratory tract. Health Phys 1966; 12: 173-207.

18 Morley C. Surfactant treatment for premature babies - a review of clinical trials. Arch Dis Child 1991; 66: 445-50. 